\title{
Operadores-escada generalizados para sistemas quânticos
}

Ladder operators for quantum systems

\author{
Hugo de Oliveira Batael ${ }^{1}$, Josimar Fernando da Silva*1, Alexandre Nogueira da Silva ${ }^{1}$, Saiara \\ Fabiana Menezes dos Santos ${ }^{1}$, Elso Drigo Filho ${ }^{1}$
}

${ }^{1}$ Universidade Estadual Paulista, Instituto de Biociências, Letras e Ciências Exatas, Câmpus São José do Rio Preto, São José do Rio Preto, SP, Brasil.

Recebido em 14 de Junho, 2017. Revisado em 16 de Agosto, 2017. Aceito em 19 de Agosto, 2017.

\begin{abstract}
A superálgebra vem sendo aplicada na resolução de diversos problemas quânticos. Ela permite, por exemplo, obter soluções para potenciais exatamente solúveis e ampliar a construção de operadores de criação e destruição. Neste trabalho, o método de construção dos operadores-escada generalizados é revisado e usado para obter os autovalores de energia e as autofunções de dois potenciais, a partícula em uma caixa unidimensional e o potencial de Rosen-Morse unidimensional.
\end{abstract}

Palavras-chave: supersimetria; mecânica quântica; shape invariance; operadores-escada generalizados; partícula em uma caixa; potencial de Rosen-Morse.

\begin{abstract}
Supersymmetry has been applied to obtain the solution of several kinds of quantum problems. For example, it allows us to solve the Schrödinger equation and to introduce the creation and annihilation operators. In this work, the method to obtain the generalized ladder operators is revised and it is used to determine the energy eigenvalues and the eigenfunctions for two potentials, a particle in a box and the one-dimensional Rosen-Morse potential.

Keywords: supersymmetry; quantum mechanics; shape invariance; ladder operators; particle in a box; RosenMorse potential.
\end{abstract}

\section{Introdução}

Em estudos envolvendo sistemas descritos pela Mecânica Quântica, podemos fazer uso do formalismo da supersimetria para a resolução da equação de Schrödinger [1,2]. Em comparação à resolução direta da equação de Schrödinger independente do tempo, este formalismo permite, por exemplo, uma simplificação dos cálculos e atacar outros tipos de problemas, como aqueles envolvendo potenciais parcialmente solúveis $[3$ 6]. Assim, essa abordagem tem motivado muitos estudos e estendido a compreensão sobre diversos sistemas quânticos (vide, por exemplo, Ref. $3[20]$ ).

O tratamento envolvendo a supersimetria em Mecânica Quântica pode ser visto como generalização do método de fatorização 1, 2]. Neste sentido, são introduzidos os operadores que fatorizam a equação de Schrödinger. Assim, ao invés de resolver uma equação diferencial de segunda ordem, é preciso resolver uma equação diferencial de primeira ordem, conhecida como equação de Riccati 21.

O formalismo da Mecânica Quântica Supersimétrica pode ser aplicado em diversos contextos. Em especial, ele é útil para estudar potenciais exatamente solúveis 11 37 7 16 e parcialmente solúveis $[1-6,18$. Em potenciais exatamente solúveis, o formalismo oferece duas vias de solução: através da Hierarquia de Hamiltonianos $\left[\begin{array}{l}1,3,7, \\ \text {, }\end{array}\right.$

*Endereço de correspondência: josimarfsilva@sjrp.unesp.br
11. e usando a chamada Shape Invariance do potencial [1, $1,2,10,12,16$.

Em potenciais para os quais não é possível determinar soluções analíticas exatas, a superálgebra ainda é útil como suporte nos métodos aproximativos, tais como perturbativo 1], aproximação WKB [13] e variacional 2, 3.18. Recentemente, a superálgebra tem sido empregada para auxiliar a solução da equação de Fokker-Planck 18 20 e para a obtenção de funções de onda teste (ansatz) para o estudo de interações intermoleculares [17, 19].

Problemas que envolvem sistemas quânticos em que os potenciais tratados são invariantes na sua forma funcional por transformações de supersimetria (shape invariant) podem ser resolvidos via superálgebra usando os operadores-escada generalizados 90,12 que atuam simultaneamente na coordenada espacial e no parâmetro da shape invariance.

É oportuno lembrar que a construção de operadoresescada permite determinar estados coerentes 22 25] para os sistemas estudados. Em termos didáticos, os estados coerentes são discutidos na referência [26], enquanto a aplicação deste formalismo pode ser vista, por exemplo, nas referências [27] e [28]. Esses estados encontram aplicação em diferentes contextos, sendo particularmente importantes em óptica quântica 29 30.

Neste trabalho é feito uma revisão do formalismo que leva a construção dos operadores de criação e des- 
truição para potenciais shape invariant, incluindo detalhes algébricos normalmente omitidos nos textos técnicos que tratam do assunto. Depois, são usados os operadoresescada generalizados para obter os autovalores de energia e as autofunções para dois exemplos particulares, a partícula em uma caixa unidimensional e o potencial de Rosen-Morse unidimensional. A partícula em uma caixa constitui um exemplo peculiar, pois não exibe uma invariância explícita, sendo necessária uma discussão mais aprofundada desse caso. O potencial de Rosen-Morse, por seu turno, embora tenha solução conhecida, não conta ainda com a descrição dos operadores-escada indicada aqui.

Embora o formalismo apresentado aqui se concentre em problemas unidimensionais, ele pode ser diretamente estendido para sistemas com mais dimensões.

Na próxima seção, a metodologia adotada é apresentada. Em seguida, a metodologia é aplicada ao problema da partícula em uma caixa [2,12,31]. Depois, um caso particular do potencial de Rosen-Morse unidimensional $\left(V(x) \propto-\operatorname{sech}^{2}(x)\right) 710$ é estudado. Por fim, na última seção, são indicadas as conclusões a cerca do formalismo adotado.

\section{Metodologia}

Consideremos um sistema quântico descrito por um potencial e cujo Hamiltoniano associado pode ser fatorizado em termos de dois operadores de primeira ordem [2]:

$$
H=-\frac{d^{2}}{d x^{2}}+V(x)=A^{+} A^{-}+E_{0},
$$

nessa expressão $H$ é o Hamiltoniano do sistema, $A^{+}$e $A^{-}$ são chamados de operadores bosônicos e $E_{0}$ é o autovalor do nível mais baixo de energia do Hamiltoniano $H$. Por simplificação adotamos $\hbar=2 m=1$.

Os operadores bosônicos são definidos como:

$$
A^{+}=-\frac{d}{d x}+W(x, a)
$$

e

$$
A^{-}=\frac{d}{d x}+W(x, a),
$$

sendo $W(x, a)$ chamado de superpotencial, dado em função da variável de posição e de um conjunto de parâmetros $(a)$ presentes no potencial. Por razões didáticas e de apresentação, os exemplos tratados nesse trabalho possui apenas um parâmetro, $a$. Substituindo as equações (2) e (3) na equação(1), obtemos uma equação de Ricatti [21] para o superpotencial $W(x, a)$ :

$$
W^{2}(x, a)-\frac{d W(x, a)}{d x}=V(x)-E_{0} .
$$

Conhecendo o superpotencial, os companheiros supersimétricos são construídos através dos operadores bosônicos definidos nas equações (2) e (3):

$$
\begin{aligned}
H_{0} & =A^{+} A^{-}=-\frac{d^{2}}{d x^{2}}+W^{2}-\frac{d W}{d x}= \\
& -\frac{d^{2}}{d x^{2}}+V_{0}(x)=H-E_{0}
\end{aligned}
$$

$\mathrm{e}$

$$
H_{1}=A^{-} A^{+}=-\frac{d^{2}}{d x^{2}}+W^{2}+\frac{d W}{d x}=-\frac{d^{2}}{d x^{2}}+V_{1}(x) .
$$

Os Hamiltonianos $H_{0}$ e $H_{1}$ possuem o mesmo espectro de energia, com exceção do estado fundamental de $H_{0}$, para o qual não há correspondência no espectro de $H_{1}[2]$.

Notamos na equação (5) que $H_{0}$ é definido como Hamiltoniano original $(H)$ subtraído da energia do estado fundamental $\left(E_{0}\right)$. Desse modo, a função de onda para o estado fundamental, $\psi_{0}$, é obtida pela equação de autovalor, dada por:

$$
H_{0} \psi_{0}\left(x, a_{0}\right)=\left(H-E_{0}\right) \psi_{0}\left(x, a_{0}\right)=A^{+} A^{-} \psi_{0}\left(x, a_{0}\right)=0 .
$$

Para que a expressão (7) seja satisfeita é suficiente que o operador de destruição aplicado à autofunção seja igual à zero, ou seja, $A^{-} \psi_{0}\left(x, a_{0}\right)=0$. Dessa forma, a função de onda para o estado fundamental, a menos da constante de normalização, é dada por:

$$
\psi_{0}\left(x, a_{0}\right) \propto \exp \left(-\int_{x} W\left(\bar{x}, a_{0}\right) d \bar{x}\right) .
$$

Seguindo na construção dos operadores-escada ou de criação e destruição é necessário que o potencial a ser estudado seja shape invariant [1,2], ou seja, satisfaça a seguinte condição:

$$
R\left(a_{0}\right)=H_{1}-H_{0}=V_{1}\left(x, a_{0}\right)-V_{0}\left(x, a_{1}\right) .
$$

Nesse caso, a diferença entre os potenciais $V_{1}\left(x, a_{0}\right)$ e $V_{0}\left(x, a_{1}\right)$ não depende da coordenada espacial, ou seja, o resíduo $R\left(a_{0}\right)$ depende apenas do parâmetro $a_{0}$.

A invariância na forma funcional (shape invariance) pode envolver diferentes transformações nos parâmetros 32, entre elas, a translação, cujos parâmetros são relacionados da seguinte forma:

$$
a_{n}=a_{0}+\eta n,
$$

onde $\eta$ é o parâmetro de translação e o parâmetro $a_{n}$ é obtido transladando $a_{0} n$ vezes.

Para os potenciais que são shape invariant e cujos parâmetros estão relacionados por translação é possível definir os operadores-escada $[2,12]$ como:

$$
B_{+}\left(a_{0}\right)=A^{+}\left(a_{0}\right) T^{+}\left(a_{0}\right)=A^{+} \exp \left(\eta \frac{\partial}{\partial a_{0}}\right)
$$

$$
B_{-}\left(a_{0}\right)=T^{-}\left(a_{0}\right) A^{-}\left(a_{0}\right)=\exp \left(-\eta \frac{\partial}{\partial a_{0}}\right) A^{-},
$$


onde $A^{+}$e $A^{-}$são os operadores bosônicos, equações (2) e (3), e $T^{+}\left(a_{0}\right)$ e $T^{-}\left(a_{0}\right)$ são os operadores de translação no parâmetro.

Os operadores-escada $B_{ \pm}$, correspondem a uma generalização dos operadores criação e destruição [2]. Neste sentido, a função de onda para o estado fundamental $\psi_{0}$ é obtida aplicando o operador destruição ao estado fundamental:

$$
B_{-}\left(a_{0}\right) \psi_{0}\left(x, a_{0}\right)=T^{-}\left(a_{0}\right) A^{-}\left(a_{0}\right) \psi_{0}\left(x, a_{0}\right)=0 .
$$

Dessa forma, é suficiente que $A^{-}\left(a_{0}\right) \psi_{0}\left(x, a_{0}\right)=0$ o que conduz a um resultado similar ao obtido na equação (8):

$$
\psi_{0}\left(x, a_{0}\right) \propto \exp \left(-\int_{x} W\left(\bar{x}, a_{0}\right) d \bar{x}\right) .
$$

Na sequência, é importante identificar a seguinte relação entre os resíduos $R\left(a_{n}\right)$ e os operadores de translação [2]:

$$
R\left(a_{n}\right)=T^{+}\left(a_{0}\right) R\left(a_{n-1}\right) T^{-}\left(a_{0}\right),
$$

ou ainda,

$$
R\left(a_{n}\right) B_{+}\left(a_{0}\right)=B_{+}\left(a_{0}\right) R\left(a_{n-1}\right) .
$$

A relação da equação 16 é verificada devido à independência de $R\left(a_{n}\right)$ em relação à variável espacial, o que implica que os operadores bosônicos e $R\left(a_{n}\right)$ comutem entre si.

O próximo passo do processo é a obtenção da relação de comutação entre $H_{0}$ e o operador $B_{+}^{n}\left(a_{0}\right)$, para isso inicialmente temos,

$$
\begin{aligned}
{\left[H_{0}, B_{+}^{n}\left(a_{0}\right)\right] } & =\left[B_{+}\left(a_{0}\right) B_{-}\left(a_{0}\right), B_{+}^{n}\left(a_{0}\right)\right] \\
& =B_{+}\left(a_{0}\right) B_{-}\left(a_{0}\right) B_{+}^{n}\left(a_{0}\right) \\
& -B_{+}^{n}\left(a_{0}\right) B_{+}\left(a_{0}\right) B_{-}\left(a_{0}\right) .
\end{aligned}
$$

Lembrando que o Hamiltoniano da equação (7) pode ser escrito em termos dos operadores $B_{+}\left(a_{0}\right)$ e $B_{-}\left(a_{0}\right)$ da seguinte maneira:

$$
\begin{aligned}
H_{0} & =B_{+}\left(a_{0}\right) B_{-}\left(a_{0}\right) \\
& =A^{+}\left(a_{0}\right) T^{+}\left(a_{0}\right) T^{-}\left(a_{0}\right) A^{-}\left(a_{0}\right) \\
& =A^{+}\left(a_{0}\right) A^{-}\left(a_{0}\right),
\end{aligned}
$$

a relação de comutação da equação 17 pode ser reescrita por:

$$
\begin{aligned}
& {\left[H_{0}, B_{+}^{n}\left(a_{0}\right)\right]=B_{+}\left(a_{0}\right)\left[B_{-}\left(a_{0}\right) B_{+}\left(a_{0}\right) B_{+}^{n-1}\left(a_{0}\right)\right.} \\
& \left.-B_{+}^{n-1}\left(a_{0}\right) B_{+}\left(a_{0}\right) B_{-}\left(a_{0}\right)\right] \\
& =B_{+}\left(a_{0}\right)\left[H_{1} B_{+}^{n-1}\left(a_{0}\right)-B_{+}^{n-1}\left(a_{0}\right) H_{0}\right] .
\end{aligned}
$$

Usando a definição:

$$
\left[H_{0}, B_{+}^{n-1}\left(a_{0}\right)\right]=H_{0} B_{+}^{n-1}\left(a_{0}\right)-B_{+}^{n-1}\left(a_{0}\right) H_{0},
$$

a equação 19 pode ser escrita como

$$
\begin{aligned}
& {\left[H_{0}, B_{+}^{n}\left(a_{0}\right)\right]=B_{+}\left(a_{0}\right)\left[H_{1} B_{+}^{n-1}\left(a_{0}\right)\right.} \\
& \left.-H_{0} B_{+}^{n-1}\left(a_{0}\right)+\left[H_{0}, B_{+}^{n-1}\left(a_{0}\right)\right]\right] .
\end{aligned}
$$

No lado direito da equação 21 os termos $H_{1} B_{+}^{n-1}\left(a_{0}\right)-$ $H_{0} B_{+}^{n-1}\left(a_{0}\right)$ remetem a relação entre os Hamiltonianos encontrada na equação (9), deste modo, obtemos:

$$
\begin{aligned}
& {\left[H_{0}, B_{+}^{n}\left(a_{0}\right)\right]=B_{+}\left(a_{0}\right)\left[R\left(a_{0}\right) B_{+}^{n-1}\left(a_{0}\right)\right.} \\
& \left.+\left[H_{0}, B_{+}^{n-1}\left(a_{0}\right)\right]\right] .
\end{aligned}
$$

A partir da equação (16), a equação 22 pode ser escrita da seguinte maneira

$$
\left[H_{0}, B_{+}^{n}\left(a_{0}\right)\right]=R\left(a_{1}\right) B_{+}^{n}\left(a_{0}\right)+B_{+}\left(a_{0}\right)\left[H_{0}, B_{+}^{n-1}\left(a_{0}\right)\right] .
$$

Repetindo um procedimento similar ao adotado para chegar a equação 23, a relação de comutação $\left[H_{0}, B_{+}^{n-1}\left(a_{0}\right)\right]$ é dada por:

$$
\begin{aligned}
& {\left[H_{0}, B_{+}^{n-1}\left(a_{0}\right)\right]=R\left(a_{1}\right) B_{+}^{n-1}\left(a_{0}\right)} \\
& +B_{+}\left(a_{0}\right)\left[H_{0}, B_{+}^{n-2}\left(a_{0}\right)\right] .
\end{aligned}
$$

Substituindo a equação (24) na equação (23), temos:

$$
\begin{aligned}
& {\left[H_{0}, B_{+}^{n}\left(a_{0}\right)\right]=R\left(a_{1}\right) B_{+}^{n}\left(a_{0}\right)} \\
& +R\left(a_{2}\right) B_{+}^{n}\left(a_{0}\right)+B_{+}^{2}\left(a_{0}\right)\left[H_{0}, B_{+}^{n-2}\left(a_{0}\right)\right] .
\end{aligned}
$$

Este procedimento pode ser repetido $n$ vezes até que o comutador do lado direito da equação 25 seja igual à zero, isto é, $\left[H_{0}, B_{+}^{n-n}\left(a_{0}\right)\right]=0$. Sendo assim, obtemos:

$$
\left[H_{0}, B_{+}^{n}\left(a_{0}\right)\right]=\left[\sum_{i=0}^{n} R\left(a_{i}\right)\right] B_{+}^{n}\left(a_{0}\right) .
$$

Aplicando o comutador na função de onda para o estado fundamental e notando que para esse estado $H_{0} \psi_{0}\left(a_{0}, x\right)=0$, temos:

$$
H_{0} B_{+}^{n}\left(a_{0}\right) \psi_{0}\left(a_{0}, x\right)=\left[\sum_{i=0}^{n} R\left(a_{i}\right)\right] B_{+}^{n}\left(a_{0}\right) \psi_{0}\left(a_{0}, x\right) .
$$

Percebe-se que a equação (27) é uma equação de autovalor para o operador $H_{0}$, onde $B_{+}^{n}\left(a_{0}\right) \psi_{0}\left(a_{0}, x\right)$ e $\sum_{i=0}^{n} R\left(a_{i}\right)$ são as autofunções e os autovalores de energia, respectivamente. Desse modo, temos que as autofunções e os autovalores de energia do Hamiltoniano original são dados por: 


$$
E_{n}=E_{0}+\sum_{i=0}^{n} R\left(a_{i}\right)
$$

e

$$
\psi_{n}\left(a_{0}, x\right)=B_{+}^{n}\left(a_{0}\right) \psi_{0}\left(a_{0}, x\right),
$$

onde $E_{0}$ é a constante subtraída do Hamiltoniano original para definir $H_{0}$ (equação (5) ) e corresponde ao autovalor do estado fundamental do problema tratado.

\section{Partícula numa caixa unidimensional}

O formalismo desenvolvido na seção anterior pode ser aplicado a qualquer potencial quântico que seja shape invariant. Um caso trivial é o oscilador harmônico onde o parâmetro de translação é nulo 12 .

Um exemplo mais elaborado do uso do método dos operadores-escada para determinar as soluções da equação de Schrödinger é o sistema composto por uma partícula confinada numa caixa unidimensional 12 . O potencial que descreve esse sistema é dado por:

$$
V_{P C}(x)=\left\{\begin{array}{cc}
\infty, & x<-\frac{\pi}{2} \\
0, & -\frac{\pi}{2}<x<\frac{\pi}{2}, \\
\infty, & x>\frac{\pi}{2}
\end{array}\right.
$$

sendo que os extremos do poço de potencial estão localizados em $-\frac{\pi}{2}$ e $\frac{\pi}{2}$. Esses valores foram escolhidos por simplicidade de cálculo.

Para esse sistema, o Hamiltoniano dentro da caixa é dado por:

$$
H_{P C}=-\frac{d^{2}}{d x^{2}}
$$

onde $H_{P C}$ é o Hamiltoniano original.

Para a construção da hierarquia de Hamiltonianos, é necessária a fatorização do Hamiltoniano original [11]. Assim, obtemos o primeiro termo constituinte da hierarquia,

$$
H_{0}=H_{P C}-E_{0}=-\frac{d^{2}}{d x^{2}}+V_{0}(x),
$$

sendo $E_{0}$ o autovalor do estado fundamental do problema.

Neste caso, o superpotencial obtido de modo à fatorizar o Hamiltoniano $H_{0}$ é conhecido [11,12] e dado por:

$$
W(x)=\operatorname{tg}(x) .
$$

Com este superpotencial, os operadores bosônicos são

$$
A^{ \pm}=\mp \frac{d}{d x}+\operatorname{tg}(x)
$$

A partir das equações (33) e (34) a fatorização do Hamiltoniano é escrita como:

$$
H_{0}=H_{P C}-E_{0}=-\frac{d^{2}}{d x^{2}}+t g^{2}(x)-\sec ^{2}(x) .
$$

Utilizando na expressão 35 e a relação trigonométrica $\operatorname{tg}^{2}(x)-\sec ^{2}(x)=-1$, obtemos que:

$$
H_{0}=-\frac{d^{2}}{d x^{2}}-1
$$

o que permite a obtenção do autovalor de energia do estado fundamental do sistema, $E_{0}=1$.

A fim de verificar se esse potencial é shape invariant obtemos o companheiro supersimétrico de $H_{0}$ que é dado por:

$$
H_{1}=-\frac{d^{2}}{d x^{2}}-V_{1}(x)=-\frac{d^{2}}{d x^{2}}+\frac{2}{\cos ^{2}(x)}-1 .
$$

Este problema é especialmente interessante, pois, em uma primeira análise, as formas obtidas para os companheiros supersimétricos, $H_{0}$ e $H_{1}$, indicam que este potencial não é shape invariant, uma vez que $V_{0}=-1 \mathrm{e}$ $V_{1}=\frac{2}{\cos ^{2}(x)}-1$. Entretanto, uma análise mais profunda permite identificar uma invariância escondida 12$]$ neste caso.

Construindo a forma geral do superpotencial na hierarquia de hamiltonianos [11,12 obtemos:

$$
W_{n}(x)=n \operatorname{tg}(x),
$$

onde $n$ é um número natural diferente de zero, $(n=$ $1,2,3, \cdots)$. A hierarquia fornece o enésimo termo da superfamília de potencias e que $E_{n}^{1}=n^{2}$,

$$
V_{n}-E_{0}^{(n)}=\frac{n(n-1)}{\cos ^{2}(x)}-n^{2} .
$$

Para identificar a shape invariance escondida neste problema é necessário explicitar a dependência dos potenciais com os parâmetros dos problemas. Assim, reescrevendo a hierarquia em termos de um parâmetro $a_{0}$, obtemos um caso particular do potencial de Infeld-Hull do tipo E como discutido nas referências 12,33 :

$$
W\left(x, a_{0}\right)=a_{0} \operatorname{tg}(x) .
$$

A fatorização com o superpotencial da equação 40 leva ao Hamiltoniano:

$$
H_{0}=-\frac{d^{2}}{d x^{2}}-V_{0}(x)=-\frac{d^{2}}{d x^{2}}+\frac{a_{0}\left(a_{0}-1\right)}{\cos ^{2}(x)}-a_{0}^{2} .
$$

O companheiro supersimétrico de $H_{0}$, é escrito então:

$$
H_{1}=-\frac{d^{2}}{d x^{2}}-V_{1}(x)=-\frac{d^{2}}{d x^{2}}+\frac{a_{0}\left(a_{0}+1\right)}{\cos ^{2}(x)}-a_{0}^{2} .
$$

É importante notar que fazendo $a_{0}=1$ no hamiltoniano da expressão 41), o hamiltoniano original (equação (36)) é recuperado.

Após a obtenção dos companheiros supersimétricos, o próximo passo é usar a expressão (9) para verificar a shape invariance do potencial. Nesse caso, temos que: 


$$
R\left(a_{1}\right)=\left[\frac{a_{0}\left(a_{0}+1\right)}{\cos ^{2}(x)}-a_{0}^{2}\right]-\left[\frac{a_{1}\left(a_{1}-1\right)}{\cos ^{2}(x)}-a_{1}^{2}\right] .
$$

Para que $R\left(a_{1}\right)$ seja independente de $x$, a seguinte relação precisa ser observada,

$$
a_{1}=a_{0}+1 .
$$

Assim, da relação 10 obtemos que $\eta=1$. Deste modo, o resíduo obtido vale:

$$
R\left(a_{1}\right)=a_{1}^{2}-a_{0}^{2}=2 a_{0}+1 .
$$

Generalizando o resíduo (equação 45 ), fazendo $a_{k}=$ $a_{0}+\eta k$, encontramos:

$$
\begin{aligned}
R\left(a_{k}\right) & =a_{k}^{2}-a_{k-1}^{2} \\
& =\left(a_{0}+k\right)^{2}-\left[a_{0}+(k-1)\right]^{2} \\
& =2 a_{0}+2 k-1,
\end{aligned}
$$

onde $\eta=1$. Em seguida, podemos determinar os níveis de energia usando a equação (28):

$E_{n}=E_{0}+\sum_{k=1}^{n} R\left(a_{k}\right)=1+\sum_{k=1}^{n}\left(2 a_{0}+2 k-1\right)=n^{2}+2 a_{0} n+1$

com $a_{0}=1$ como identificado anteriormente. Esses autovalores são os mesmos obtidos por outros métodos [2]. $\mathrm{Na}$ sequência, obtemos os operadores-escada de acordo como foi apresentado nas equações (11) e 12$)$ :

$$
B_{+}\left(a_{0}\right)=\left[-\frac{d}{d x}+t g(x)\right] \exp \left(\eta \frac{\partial}{\partial a_{0}}\right)
$$

$\mathrm{e}$

$$
B_{-}\left(a_{0}\right)=\exp \left(-\eta \frac{\partial}{\partial a_{0}}\right)\left[\frac{d}{d x}+t g(x)\right]
$$

as autofunções para este potencial podem ser obtidas usando os operadores das equações (48) e (49).

Utilizando o operador $B_{-}\left(a_{0}\right)$ (equação 49 ) na função do estado fundamental, obtemos:

$$
B_{-}\left(a_{0}\right) \psi_{P C_{0}}\left(x, a_{0}\right)=0,
$$

o que permite encontrar a autofunção para o estado fundamental de uma partícula confinada em uma caixa unidimensional $\left(\psi_{P C_{0}}\left(x, a_{0}\right)\right)$ :

$$
\psi_{P C_{0}}\left(x, a_{0}\right)=N_{0} \exp \left[-\int_{x} a_{0} \operatorname{tg}(\bar{x}) d \bar{x}\right]=N_{0} \cos ^{a_{0}}(x),
$$

sendo $N_{0}$ a constante de normalização.

Para o caso em que $a_{0}=1$, temos:

$$
\psi_{P C_{0}}(x)=N_{0} \cos (x)
$$

Para o primeiro estado excitado e $a_{0}=1$, a seguinte autofunção é encontrada:

$$
\psi_{P C_{1}}(x)=N_{1} \cos (2 x),
$$

onde $N_{1}$ é a constante de normalização para este estado.

Por fim, usando as equações (29), 48) e 51 obtemos a função de onda para o enésimo estado excitado:

$$
\begin{aligned}
& \psi_{P C_{n}}\left(x, a_{0}\right) \propto\left[\left(-\frac{d}{d x}+t g(x)\right)\right. \\
& \left.\times \exp \left(\eta \frac{\partial}{\partial a_{0}}\right)\right]\left.^{n} \cos ^{a_{0}}(x)\right|_{a_{0}=1},
\end{aligned}
$$

e os autovalores de energia são dados pela equação (47),

$$
E_{n}=n^{2}+\left.\left(2 a_{0} n+1\right)\right|_{a_{0}=1} .
$$

\section{Potencial de Rosen-Morse}

Usando o formalismo descrito anteriormente é possível determinar os operadores-escada para qualquer potencial shape invariant. Outro exemplo ilustrativo do formalismo introduzido e ainda não explorado na literatura é o potencial de Rosen-Morse [34:

$$
V_{R M}(\alpha y)=-V_{a} \operatorname{sech}^{2}(\alpha y)+V_{b} \tanh (\alpha y),
$$

onde $\alpha, V_{a}$ e $V_{b}$ são constantes. O potencial unidimensional de Rosen-Morse estudado aqui, se restringe a um caso particular em que: $V_{b}=0$ e $\alpha y=x$. Dessa maneira, reescrevemos a equação 56 como:

$$
V_{R M}(x)=-V_{a} \operatorname{sech}^{2}(x) .
$$

O Hamiltoniano para este caso pode ser escrito de forma conveniente como:

$$
H_{R M}=-\frac{d^{2}}{d x^{2}}+V_{R M}(x)=-\frac{d^{2}}{d x^{2}}-V_{a} \operatorname{sech}^{2}(x)
$$

Fatorizando o Hamiltoniano (equação (58)) da mesma maneira feita na equação (1) temos que definir os operadores bosônicos, ou seja, obter um superpotencial $W_{0}(x)$ que fatorize $H_{0}$. Um superpotencial viável é:

$$
W_{0}(x)=\beta_{0} \tanh (x),
$$

onde $\beta_{0}$ é uma constante. Assim, os operadores bosônicos para este caso são definidos como:

$$
A^{ \pm}=\mp \frac{d}{d x}+\beta_{0} \tanh (x) .
$$

A fatorização deste caso é escrita como:

$$
H_{R M}-E_{0}=A^{+} A^{-}=-\frac{d^{2}}{d x^{2}}+\beta_{0}^{2} \tanh ^{2}(x)-\beta_{0}^{2} \operatorname{sech}^{2}(x) .
$$


Usando a relação $\operatorname{sech}^{2}(x)+\tanh ^{2}(x)=1$, a equação 61 pode ser expressa de uma maneira conveniente por:

$$
H_{0}=H_{R M}-E_{0}=-\frac{d^{2}}{d x^{2}}+\beta_{0}^{2}\left(1-\operatorname{sech}^{2}(x)\right)-\beta_{0}^{2} \operatorname{sech}^{2}(x) .
$$

Para determinar a constante é preciso igualar a equação (62) com o Hamiltoniano original (equação (58)), isto é,

$$
\begin{aligned}
-\frac{d^{2}}{d x^{2}}-V_{a} \operatorname{sech}^{2}(x)-E_{0}= & -\frac{d^{2}}{d x^{2}}+\beta_{0}^{2}\left(1-\operatorname{sech}^{2}(x)\right) \\
& -\beta_{0}^{2} \operatorname{sech}^{2}(x) .
\end{aligned}
$$

A equação 63 é verdadeira quando:

$$
\beta_{0}=\frac{-1+\sqrt{1+4 V_{a}}}{2} \text { onde } V_{a}>0
$$

e

$$
E_{0}=-\beta_{0}^{2}
$$

É oportuno observar que $\beta_{0}$ foi escolhido de forma que $\beta_{0}>0$ para todo $V_{a}>0$. Essa condição é importante, pois possibilita a existência de estados ligados e que as funções de onda obtidas adiante sejam normalizáveis.

Observa-se que o potencial $V_{0}(x)$ nesse caso, é definido como:

$$
V_{0}(x)=\beta_{0}^{2}\left(1-\operatorname{sech}^{2}(x)\right)-\beta_{0} \operatorname{sech}^{2}(x) .
$$

Seguindo a metodologia apresentada, é necessário verificar se esse potencial é shape invariant. Assim, usando a equação 60 determinamos o companheiro supersimétrico de $H_{0}$ :

$$
\begin{aligned}
H_{1} & =A^{-} A^{+} \\
& =-\frac{d^{2}}{d x^{2}}+\beta_{1}^{2} \tanh ^{2}(x)+\beta_{1}^{2} \operatorname{sech}^{2}(x) \\
& =-\frac{d^{2}}{d x^{2}}+\beta_{1}^{2}\left(1-\operatorname{sech}^{2}(x)\right)+\beta_{1}^{2} \operatorname{sech}^{2}(x) .
\end{aligned}
$$

Comparando a equação 66 com a equação 67 percebese que o superpotencial associado com $H_{1}$ pode ser escrito como:

$$
W_{1}(x)=\beta_{1} \tanh (x),
$$

onde $\beta_{1}$ é uma constante a ser determinada. Usando essa função, o potencial $V_{1}(x)$ é definido como:

$$
V_{1}(x)=\beta_{1}^{2}\left[1-\operatorname{sech}^{2}(x)\right]+\beta_{1} \operatorname{sech}^{2}(x) .
$$

Usando as equações (66) e 69 verificamos que o potencial original é shape invariant (equação (9)) se:

$$
\begin{aligned}
& R\left(\beta_{1}\right)=\beta_{0}^{2}\left[1-\operatorname{sech}^{2}(x)\right]+\beta_{0} \operatorname{sech}^{2}(x) \\
& -\beta_{1}^{2}\left[1-\operatorname{sech}^{2}(x)\right]-\beta_{1} \operatorname{sech}^{2}(x),
\end{aligned}
$$

para $R\left(\beta_{1}\right)$ ser independente da variável espacial o parâmetro $\beta_{1}$ tem que ser escrito como:

$$
\beta_{1}=\beta_{0}-1
$$

Fazendo uma relação entre a equação 10 e a equação (71) nota-se que o parâmetro de translação $\eta$ é -1 . Portanto, o resíduo $R\left(\beta_{1}\right)$ é:

$$
R\left(a_{1}\right)=\beta_{0}^{2}-\beta_{1}^{2}=2 \beta_{1}+1 .
$$

No geral, o resíduo (equação 72 ) é generalizado de maneira análoga a equação 10 o que implica em $\beta_{k}=$ $\beta_{0}+\eta k$, onde $\eta=-1$. Assim, $R\left(\beta_{k}\right)$ pode ser redefinido:

$$
\begin{aligned}
R\left(\beta_{k}\right) & =\beta_{k-1}^{2}-\beta_{k}^{2}=\left[\beta_{0}-(k-1)\right]^{2}-\left(\beta_{0}-k\right)^{2} \\
& =2\left(\beta_{0}-k\right)+1
\end{aligned}
$$

Usando as equações (28), 65 e (73), calculamos os níveis de energia para o potencial de Rosen-Morse estudado:

$$
\begin{aligned}
E_{n} & =-\beta_{0}^{2}+\sum_{k=1}^{n}\left[2\left(\beta_{0}-k\right)+1\right] \\
& =-\beta_{0}^{2}+\left(2 \beta_{0}+1\right) n-n(n+1) \\
& =-\left(\beta_{0}-n\right)^{2},
\end{aligned}
$$

onde $\beta_{0}$ é definido pela equação (64). Esses autovalores de energia são os mesmos encontrados por outros métodos 7, 8.

Em seguida, se obtém os operadores de criação e de destruição das definições dadas pelas equações (11) e 12), respectivamente, com o superpotencial indicado pela equação (59). Para o presente problema esses operadores são dados por:

$$
B_{+}\left(\beta_{0}\right)=\left[-\frac{d}{d x}+\beta_{0} \tanh (x)\right] \exp \left(\eta \frac{\partial}{\partial \beta_{0}}\right)
$$

$$
B_{-}\left(\beta_{0}\right)=\exp \left(-\eta \frac{\partial}{\partial \beta_{0}}\right)\left[\frac{d}{d x}+\beta_{0} \tanh (x)\right],
$$

As autofunções para o potencial original, equação (57), podem ser encontrados usando os operadores de criação (equação (75)) e destruição (equação 76 ). Utilizando o operador $\bar{B}_{-}\left(\beta_{0}\right)$ aplicado à autofunção do estado fundamental, deve-se observar que:

$$
B_{-}\left(\beta_{0}\right) \psi_{R M_{0}}\left(x, \beta_{0}\right)=0,
$$

o que permite determinar a autofunção para o estado fundamental do potencial de Rosen-Morse, $\left(\psi_{R M_{0}}\left(x, \beta_{0}\right)\right)$ :

$$
\psi_{R M_{0}}\left(x, \beta_{0}\right)=N_{0} \cosh ^{-\beta_{0}}(x),
$$


onde $N_{0}$ é a constante de normalização e $\beta_{0}$ é dado na equação 64.

$$
\psi_{R M_{1}}\left(x, \beta_{0}\right)=N_{1} \operatorname{senh}(x) \cosh ^{-\left(\beta_{0}+2\right)}(x),
$$

onde $N_{1}$ é a constante de normalização para este estado.

Por fim, usando as equações (29), 775) e (78) obtemos a função de onda para o enésimo estado excitado:

$$
\begin{aligned}
& \psi_{R M_{n}}\left(x, \beta_{0}\right) \propto\left[\left(-\frac{d}{d x}+\beta_{0} \tanh (x)\right)\right. \\
& \left.\times \exp \left(\eta \frac{\partial}{\partial \beta_{0}}\right)\right]\left.^{n} \cosh ^{-\beta_{0}}(x)\right|_{\beta_{0}=\frac{-1+\sqrt{1+4 V_{a}}}{2}}
\end{aligned}
$$

onde $V_{a}>0$ e os autovalores de energia (equação (74)) são dados por:

$$
E_{n}=-\left.\left(\beta_{0}-n\right)^{2}\right|_{\beta_{0}=\frac{-1+\sqrt{1+4 V_{a}}}{2}} .
$$

\section{Conclusões}

Neste trabalho foram introduzidos os operadores-escada generalizados, originários da aplicação do formalismo da Supersimetria em Mecânica Quântica. O formalismo foi introduzido com detalhes algébricos e usado para obtenção das autofunções e dos autovalores de energia, para a partícula em uma caixa e um caso particular do potencial de Rosen-Morse.

Para os dois casos estudados a shape invariance foi identificada, o que viabilizou a construção dos operadores-escada generalizados. No caso da partícula numa caixa, diferentemente do que ocorre para o potencial de Rosen-Morse, a shape invariance não se deu de forma direta. Ela só pode ser identificada após a introdução de um parâmetro fixado a posteriori, o que foi indicado com uma invariância escondida.

Os resultados apresentados mostram uma maneira alternativa de construção dos operadores-escada em Mecânica Quântica. Uma vez que quase todos os potenciais exatamente solúveis são shape invariant a abordagem proposta generaliza o tratamento via operadores-escada para um número maior de problemas. Alguns desses problemas já foram tratados na literatura [2, 12, como o caso da partícula em uma caixa. Entretanto, até onde podemos perceber, o caso particular do potencial de Rosen-Morse estudado aqui ainda não havia sido discutido através dessa abordagem até o presente trabalho.

Os resultados obtidos aqui podem ser usados em diferentes contextos, em especial, na construção de estados coerentes, o que amplia o interesse pela abordagem apresentada. O formalismo discutido também pode ser visto como um método alternativo de obtenção da solução da equação Schrödinger.

\section{Agradecimentos}

Os autores agradecem a Coordenação de Aperfeiçoamento de Pessoal de Nível Superior (CAPES) e a Fundação de Amparo à Pesquisa do Estado de São Paulo (FAPESP) pelo suporte financeiro parcial ao projeto.

\section{Referências}

[1] F. Cooper, A. Khare and U. Sukhatme, Phys. Rep. 251, 267 (1995).

[2] E. Drigo Filho, Supersimetria Aplicada à Mecânica Quântica: Estudo da Equação de Schrödinger (Editora UNESP, São Paulo, 2009).

[3] E. Drigo Filho and R.M. Ricotta, Mod. Phys. Lett. A 10, 1613 (1995).

[4] E. Drigo Filho and R.M. Ricotta, Phys. Lett. A 269, 269 (2000).

[5] D. Mikulski, J. Konarski, K. Eder, M. Molski and S. Kabaciński, J. Math. Chem. 53, 2018 (2015).

[6] J.C.B. Araujo, G.R.P. Borges and E. Drigo Filho, Rev. Bras. Ens. Fis. 28, 41 (2006).

[7] C.V. Sukumar, J. Phys. A: Math. Gen. 18, 2917 (1985).

[8] R. Dutt, A. Khare and U.P. Sukhatme, Am. J. Phys. 56, 163 (1988).

[9] N.A. Alves and E. Drigo Filho, J. Phys. A: Math. Gen. 21, 3215 (1988).

[10] G. Levai, J. Phys. A: Math. Gen. 22, 689 (1989).

[11] E. Drigo Filho, Rev. Bras. Ens. Fis. 20, 258 (1990).

[12] E. Drigo Filho and M.R. Ricotta, J. Phys. A: Math. Gen. 37, 10057 (2004).

[13] C.S. Jia, J.Y. Wang, S. He, and L.T. Sun, J. Phys. A: Math. Gen. 33, 6993 (2000).

[14] S.W. Qian, B.W. Huang and Z.Y. Gu, New J. Phys. 4, 13 (2002).

[15] B. Bagchi, A. Banerjee, C. Quesne and V.M. Tkachuk, J. Phys. A: Math. Gen. 38, 2929 (2005).

[16] A. Ganguly and L.M. Nieto, J. Phys. A: Math. Theor. 40, 7265 (2007).

[17] C.S. dos Santos, E. Drigo Filho and R.M. Ricotta, Int. J. Quantum Chem. 115, 765 (2015).

[18] G.R.P. Borges, E. Drigo Filho and R.M. Ricotta, Physica A 389, 3892 (2010).

[19] F.R. Silva and E. Drigo Filho, Chem. Phys. Lett. 498, 198 (2010).

[20] F. Polotto, M.T. Araujo and E. Drigo Filho, J. Phys. A: Math. Theor. 43, 15207 (2010).

[21] S. Bittanti, A.J. Laub and J.C. Willems, The Riccati Equation (Springer - Verlag, New York, 2012).

[22] E. Merzbacher, Quantum Mechanics (Wiley, New York, 1997).

[23] R. Dick, Advanced Quantum Mechanics - Materials and Photons (Springer - Verlag, New York, 2012).

[24] A.A. Raduta, Nuclear Structure with Coherent States (Springer International Publishing, Switzerland, 2015).

[25] R. Borrelli and M.F. Gelin, Chem. Phys. 481, 91 (2016).

[26] I. Silva, Rev. Bras. Ens. Fis. 37, 4204 (2015).

[27] M. Novaes, Rev. Bras. Ens. Fis. 24, 437 (2002).

[28] C. Valverde, A.N. Castro, E.P. Santos e B. Baseia, Rev. Bras. Ens. Fis. 37, 2311 (2015). 
[29] W.M. Zhang, D.H. Feng and R. Gilmore, Rev. Mod. Phys. 62, 867 (1990).

[30] P.L. Knight and L. Allen, Concepts of Quantum Optics (Pergamon, Oxford, 2013).

[31] D.J. Griffiths, Introduction to Quantum Mechanics (Pearson Prentice Hall, New Jersey, 2004).

[32] A.B. Balantekin, Phys. Rev. A 57, 4188 (1998).

[33] L. Infeld and T.E. Hull, Rev. Mod. Phys. 23, 21 (1951).

[34] A.N. Ikot and L.E. Akpabio, App. Phys. Res. 2, 202 (2010). 